\title{
ISOMORPHS OF A CLASS OF QUEUEING SYSTEMS
}

\author{
A. GHOSAL and SUDHIR MADAN \\ Operational Research University \\ Group of \\ CSIR, New Delhi Delhi
}

(Received September 1, 1978)

ABSTRACT. Restricted isomorphism between two queueing systems implies that they have equivalent distribution function for at least one (but not all) output elements (e.g. waiting time, queue size, idle time, etc.). Quasi-isomorphism implies an approximate equivalence. Most of the single-server queueing systems can be approximated by a quasi-isomorphic system which has a gamma inter-arrival and gamma service distributions $\left(\mathrm{E}_{\mathrm{p}} / \mathrm{E}_{\mathrm{q}} / 1\right)$.

This paper deals with the derivation of simpler isomorphs (in quasi-restricted sense) for such gamma-gamma $\left(E_{p} / E_{q} / 1\right)$ systems.

KEY WORDS AND PHRASES. Isomorph, restricted isomorph, quasi-restricted (2.R.) isomorph, waiting time, queise size, idle time, Gamma distribution.

AMS(MOS) SUBJECT CLASSIFICATION (1970) CODES.

1. INTRODUCTION.

The concept of isomorphism, in the restricted and quasi-sense, between two queueing systems was developed by Ghosal $(1977,1978)$ who also exh1bited the 
usefulness of this concept in deriving approximate solutions to practical problems. Most of the single-server queueing systems in real life are of the type GIFG/1 in which the inter-arrival time between consecutive customers follows a general probabilitydistribution function (d.f.), the service time also follows a general d.f.. The analytical solution for the d.f. of waiting time, queue size or idle time (of server) etc. is available only for a limited class of problems. Most of the studies in approximation concentrate on deriving an approximate solution of either an integral equation or a difference-differential equation. The method of isomorphs, developed by Ghosal (1977), is directed towards finding a simpler system for which the solution is known but which is equivalent to the parent system in respect of the required output property, e.g. d.f. of waiting time or d.f. of idle time or d.f. of inter-departure process, etc.. Equivalence in d.f. in one or two but not all output elements is called restricted isomorphism. If two systems have approximately equivalent (in d.f.) output elements, one may be called a quasi-isomorph of the other : if approximate equivalence relates only to one or two (but not all) of the output elements, this phenomenon is called quasi-restricted isomorphism.

Throughout this paper, whenever we refer to d.f. of an element (waiting time, queue size, idle time, etc.), we imply the equilibrium d.f. Concepts and notations of Ghosal (1977) have been used in this paper.

It has been observed by Kendall (1964), Lilliefors (1966) that any GI/G/1 can be approximated by an $\mathrm{E}_{\mathrm{p}} / \mathrm{E}_{\mathrm{q}} / 1$ system, in which the inter-arrival time $\left(\mathrm{t}_{\mathrm{n}}\right)$ follows a gamma distribution with parameter $\mathrm{p}, \mathrm{u}$.

$$
\begin{array}{r}
\operatorname{pr}\left(x<t_{n}<x+d x\right)=\left\{\lambda^{p} /(p-1) !\right\} \exp (-\lambda x) x^{p-1} d x \\
x \in(0, \infty), \lambda \geq 1:
\end{array}
$$

and the service time $\left(s_{n}\right)$ follows a Gamma distribution with parameter $q$, i.e. 


$$
\left.\operatorname{Pr}\left(x<s_{n}<x+d x\right)=\left\{\mu^{q} / q-1\right) !\right\} \exp (-\mu x) x^{q-1} d x,(x \in(0, \infty) ; \mu \geq 1)(1.2)
$$

The choice of $p, q, \lambda$ and $\mu$ may be made from experimental studies by actually studying statistical distributions of waiting time, queue size, etc.. A few practical studies, done by the authors (not reported here) lead to an interesting observation: the quasi-restricted (Q.R.) isomorph which approximates the parent system in respect of waiting time d.f. in the best possible manner may have a set of parameters different from one which approximates the parent system in respect of idle time $d . f$. in the best possible manner. Thus the best Q.R. isomorphs in respect of waiting time d.f. and idle time d.f. may have the parameter sets $\left(\mathrm{p}_{1}, \mathrm{q}_{1}, \lambda{ }_{1}, \mu_{1}\right)$ and $\left(\mathrm{p}_{2}, \mathrm{q}_{2}, \lambda_{2}, \mu_{2}\right)$ respectively $\left(\mathrm{p}_{1} \neq \mathrm{p}_{2}, \mathrm{q}_{1} \neq \mathrm{q}_{2}, \ldots\right)$.

This paper deals with the problem of determining simpler Q.R. isomorphs of type $M / M / 1$ or $M / E_{r} / 1$ for parent $E_{p} / E_{q} / 1$ systems. In practical problems we have to estimate errors in two stages of approximation: (a) while using a $\mathrm{E}_{\mathrm{p}} / \mathrm{E}_{\mathrm{q}} / 1 \mathrm{Q} \cdot \mathrm{R}$. isomorph for the parent system, (b) while using an $\mathrm{M} / \mathrm{M} / 1$ or $M / E_{r} / 1$ Q.R. isomorph for a parent $E_{p} / E_{q} / 1$. The paper concentrates on determining Q.R. isomorphs of simple forms $\left(M / M / 1\right.$ or $\left.M / E_{r} / 1\right)$ rather than on the estimation of isomorphs in two-step approximation.

\section{SOME RESULTS.}

This paper rests on the following known theorem:

THEOREM 2.1. If there are two single-server queueing systems $\left\{W^{(1)}, u^{(1)}\right\},\left\{W^{(2)}, u^{(2)}\right\}$, where $W^{(1)}$ is the waiting time of the $n$th customer, $u^{(i)}=s^{(i)}-t^{(i)}$, where $s^{(i)}$ is the service time and $t^{(i)}$ is the interarrival time between two customers $(i=1,2)$, then if $u(1)$ and $u^{(2)}$ have equivalent d.f.s, $W^{(1)}$ and $W^{(2)}$ are also equivalent in d.f. (see Lindley (1952), Ghosal (1970)).

From the above we derive the following corollary in queues which are 
isomorphic in the quasi- or quasi-restricted sense.

COROLIARY 2.1.1. If there are two single-server systems exemplified by $\left\{\mathrm{W}^{(1)}, \mathrm{u}^{(1)}\right\}$ and $\left\{\mathrm{W}^{(2)}, \mathrm{u}^{(2)}\right\}$, then if $\mathrm{u}^{(1)}$ is approximately equivalent to $u^{(2)}$ in d.f. then $W^{(1)}$ is also approximately equivalent to $W^{(2)}$ in d.f., in other words the two systems are isomorphic in the quasi-restricted sense (w.r.t. waiting time d.f.).

In our search for Q.R. isomorphs for an $E_{p} / E_{q} / 1$ system, we are to look for another system which have approximately equivalent d.f. w.r.t. u.. Let the interarrival time follow d.f. (" $\sim$ " stands for "has the probaltitty distribution")

$$
t \frown\left\{\lambda^{p} /(p-1) !\right\} \exp (-\lambda x) x^{p-1} d x,(\lambda>0: x \in(0, \infty) p>1)(2.1)
$$

and the service time (s) follows the d.f.

$$
s \frown\{\mu q /(q-1) !\} \exp (-\mu x) x^{q-1} d x,(\mu>0: x \in(0, \infty), q>1)(2.2)
$$

Let $u=s-t$ and $G(x)=\operatorname{prob}(u \leq x)$, and let its Laplace transform (L.T) be given by

$$
\psi(\theta)=\int_{-\infty}^{\infty} \exp (-\theta x) \mathrm{d} G(x),
$$

If $T(\theta)$ and $S(\theta)$ be respectively L.T.'s of $t$ and $s$, then we get

$$
\psi(\theta)=S(\theta) T(-\theta),
$$

so that in the above case

$$
\begin{aligned}
\psi(\theta) & =\left(\frac{\lambda}{\lambda-\theta}\right)^{p}\left(\frac{\mu}{\mu+\theta}\right)^{q} \\
& =\left(1-\frac{\theta}{\lambda}\right)^{-p}\left(1+\frac{\theta}{\mu}\right)^{-q}
\end{aligned}
$$




$$
\begin{aligned}
= & 1-\theta\left(\frac{q}{\mu}-\frac{p}{\lambda}\right)+\frac{\theta}{2}^{2}\left[\frac{q(q+1)}{\mu^{2}}+\frac{p(p+1)}{\lambda^{2}}-\frac{2 p q}{\lambda \mu}\right] \\
& -\frac{\theta^{3}}{6}\left[\frac{q(q+1)(q+2)}{\mu^{3}}-\frac{3 p q(q+1)}{\mu^{2} \lambda}+\frac{3 p q(p+1)}{\mu \lambda^{2}}-\frac{p(p+1)(p+2)}{\lambda^{3}}\right]+\ldots,
\end{aligned}
$$

Let there be a second system for which $S_{2}(\theta)$ is the L.T. of service time $\left(s_{2}\right)$ and $T_{2}(\theta)$ is the L.T. of interarrival time $\left(t_{2}\right)$. If $s_{2}$ follows an exponential d.f. with parameter $\mu_{2}$ and $t_{2}$ follows an exponential d.f. with parameter $\lambda_{2}$, then $\phi_{2}(\theta)$, L.T. of $u_{2}\left(=s_{2}-t{ }_{2}\right)$ is given by

$$
\begin{aligned}
\phi_{2}(\theta) & =S_{2}(\theta) T_{2}(-\theta) \\
& =\left(1-\frac{\theta}{\lambda}\right)^{-1}\left(1+\frac{\theta}{\mu_{2}}\right)^{-1} \\
& =1-\theta\left(\frac{1}{\mu_{2}}-\frac{1}{\lambda_{2}}\right)+\theta^{2}\left(\frac{1}{\mu_{2}^{2}}+\frac{1}{\lambda_{2}^{2}}-\frac{1}{\lambda_{2} \mu_{2}}\right)+\ldots
\end{aligned}
$$

If the coefficlents of $\theta$ and $\theta^{2}$ are equal in (2.4) and (2.5), we get

$$
\begin{gathered}
\frac{1}{\mu_{2}}-\frac{1}{\lambda_{2}}=\frac{q}{\mu}-\frac{p}{\lambda}, \\
2\left(\frac{1}{\mu_{2}^{2}}+\frac{1}{\lambda_{2}^{2}}-\frac{1}{\lambda_{2} \mu_{2}}\right)=\frac{q(q+1)}{\mu^{2}}+\frac{p(p+1)}{\lambda^{2}}-\frac{2 p q}{\lambda u}
\end{gathered}
$$

\begin{tabular}{|c|c|c|}
\hline Service No. & $\begin{array}{l}\text { Parameters in } \\
\text { system } 1\end{array}$ & $\begin{array}{l}\text { Parameters in the M/M/1 } \\
\text { Isomorph (System) } 2\end{array}$ \\
\hline 1. & $\begin{aligned} p & =2, q=3 \\
\lambda & =0.3, \mu=0.6\end{aligned}$ & $\begin{array}{l}\lambda_{2}=0.2150, \mu_{2}=0.3350 \\
\rho_{2}=0.6418\end{array}$ \\
\hline 2. & $\begin{array}{l}p=3, q=2 \\
\lambda=0.4, \mu=0.4\end{array}$ & $\begin{array}{l}\lambda_{2}=0.2 \\
\rho_{2}=0.50\end{array}$ \\
\hline 3. & $\begin{array}{l}p=3, q=4 \\
\lambda=0.5 \quad \mu=0.8\end{array}$ & $\begin{array}{l}\lambda_{2}=0.2874, \quad L_{2}=0.4034 \\
\rho_{2}=0.7124\end{array}$ \\
\hline
\end{tabular}

From (2.6) and (2.7) we get

$$
\frac{1}{\mu_{2}^{2}}-\frac{1}{\lambda_{2}^{2}}=\frac{q}{\mu^{2}}+\frac{p}{\lambda^{2}}
$$

The two equations (2.6) and (2.8) give a complete solution for $\mu_{2}$ and $\lambda_{2}$. We shall elucidate the solution by giving a few practical examples.

Table 2.1 
It may be observed from Table 2.1 that we do not get good approximation for the parameters of the $M / M / 1$ isomorph by equation $\mu_{2}=\mu / q, \lambda_{2}=\lambda / p$. However, by applying the approximation method as given above we can estimate difference in third moments between the parent system and the isomorph. A good approximation for traffic intensity is obtained from the $M / M / 1$ isomorph $\left(\rho_{2}=\lambda_{2} / \mu_{2}\right)$, hence probability that waiting time is zero is approximately

$$
\operatorname{Pr}(\mathrm{W}=0)=1-\rho_{2} \quad .
$$

It may be borne in mind that the traffic intensity $(\rho)$ in the $E_{p} / E_{q} / 1$ system does not enable us to determine prob $(W=0)$, as in (2.9).

3. ISOMORPHS OF M/E $\mathrm{E}_{\mathrm{k}} / 1$ TYPE.

It may be interesting to probe into the problem of determination of isomorphs of $M / E_{k} / 1$ type with respect to the waiting time d.f.. The L.T. $\phi(\theta)$ of the parent $\mathrm{E}_{\mathrm{p}} / \mathrm{E}_{\mathrm{q}} / 1$ system is given by (2.4). In the isomorph of $\mathrm{M} / \mathrm{E}_{\mathrm{k}} / 1$ type, let the interarrival time follow the d.f..

$$
\lambda_{2} \exp \left(-\lambda_{2} x\right) d x, x \in(0, \infty),
$$

and the service time follow the d.f..

$$
\left\{\mu_{2}^{k} /(k-1) !\right\} \exp \left(-\mu_{2} x\right) x^{k-1} d x,\left(\mu_{2}>0, x \in(0, \infty), k>1\right)
$$

Then the L.T. $\phi_{2}(\theta)$ of $u^{(2)}$ is given by

$$
\begin{aligned}
\phi_{2}(\theta) & =\left(1-\frac{\theta}{\lambda_{2}}\right)^{-1}\left(1+\frac{\theta}{\mu_{2}}\right)^{k} \\
& =1-\theta\left(\frac{k}{\mu_{2}}-\frac{1}{\lambda_{2}}\right)+\frac{\theta^{2}}{2}\left(\frac{k(k+1)}{\mu_{2}}+\frac{2}{\lambda_{2}^{2}}-\frac{2 k}{\lambda_{2} \mu_{2}},\right.
\end{aligned}
$$

If the coefficients of $\theta, \theta^{2}$ and $\theta^{3}$ are to be the same in (2.4) and (3.1), we get

$$
\frac{\mathrm{q}}{\mu}-\frac{\mathrm{p}}{\lambda}=\frac{\mathrm{k}}{\mu_{2}}-\frac{1}{\lambda_{2}}
$$




$$
\begin{aligned}
& \frac{q(q+1)}{\mu^{2}}+\frac{p(p+1)}{\lambda^{2}}-\frac{2 p q}{\lambda \mu}=\frac{k(k+1)}{\mu_{2}^{2}}+\frac{2}{\lambda_{2}^{2}}-\frac{2 k}{\lambda_{2} \mu_{2}}, \\
& \frac{q(q+1)(q+2)}{\mu^{3}}-\frac{p(p+1)(p+2)}{\lambda^{3}}+\frac{3 p(p+1) q}{\lambda^{2}{ }^{2}}-\frac{3 q(q+1) p}{\lambda^{2}} \\
& =\frac{k(k+1)(k+2)}{\mu_{2}^{3}}-\frac{6}{\lambda_{2}^{3}+\frac{3 k(k+1)}{\lambda_{2} \mu_{2}^{2}}-\frac{6 k}{\lambda_{2}^{2}}},
\end{aligned}
$$

From (3.2) and (3.3) we get

$$
\frac{k}{\mu_{2}^{2}}+\frac{1}{\lambda_{2}^{2}}=\frac{p}{\lambda^{2}}+\frac{q}{\mu^{2}}
$$

From (3.2) and (3.4) we get

$$
\frac{3 k^{2}+2 k}{\mu_{2}^{3}}-\frac{5}{\lambda_{2}^{3}}-\frac{3 k}{\mu_{2}^{2} \lambda}+\frac{3 k}{\lambda_{2}^{2} \mu_{2}}=\frac{3 q^{2}+2 q}{\mu^{3}}-\frac{3 p^{2}+2 p}{\lambda^{2}}+\frac{3 p q}{\lambda^{2} \mu}-\frac{3 p q}{\lambda \mu^{2}} \text {. }
$$

The three parameters of the isomorph, $k, \lambda_{2}$ and $\mu_{2}$ can be numerically solved from the set of equations (3.2) through (3.6) by method of iteration. One approach would be to select various integral values of $k$ and solve for $\lambda_{2}$ and $\mu_{2}$ and hence to estimate for what value of $k$ the difference between the right hand side and the left hand side in (3.6) is minimum.

\section{CONCLUDING REMARKS.}

It has been shown in this paper how quasi-isomorphs of the type $M / M / 1$ or $M / E_{r} / 1$ for an $E_{p} / E_{q} / 1$ can be derived on the assumption of Theorem 2.1 and Corollary 2.1.1. Some numerical experiments (which have not been reported in this paper but will be reported subsequently in detail) suggest practical utility of approximation through isomorphs. These experiments indicate the effect of approximations given in Section 2 and 3 on waiting time distributions.

There is scope for extending the approach of isomorphs in approximating waiting time distribution or queue size distribution in complicated network queues. 
ACKNOWLEDGEMENT. The authors are grateful to Dr. A. Ramachandran, DirectorGeneral, CSIR for encouragement in the work in Operations Research and Mathematical Systems.

\section{REFERENCE}

1. Ghosal, A., Some Aspects of Queueing and Storage Systems, Lecture Notes in $0 . R$. and Math. Systems Vo1. 23, Springer Verlag, New York, 1970.

2. Ghosal, A., Isomorphic Queues, Bull. Austr. Math. Soc. 17 (1977) 275-290.

3. Ghosal, A., Applied Cybernetics: its relevance to Operations Research, Gordon and Breach, New York, (see Chapter 4), 1978.

4. Kendall, David G., Some recent work and futher problems in the theory of queues, Theory of Probability and its Applications 9 (1964) 1-13.

5. Lilliefors, Hubert, W., Some confidence intervals for queues, Operations Res. 14 (1966) 723-727.

6. Lindley, D.V., The theory of queues with a single server, Proc. Comb. Phil. Soc. 48 (1952) 227-289. 


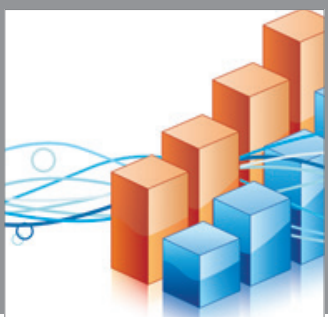

Advances in

Operations Research

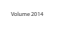

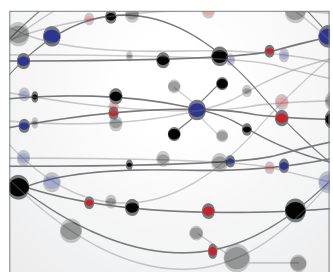

\section{The Scientific} World Journal
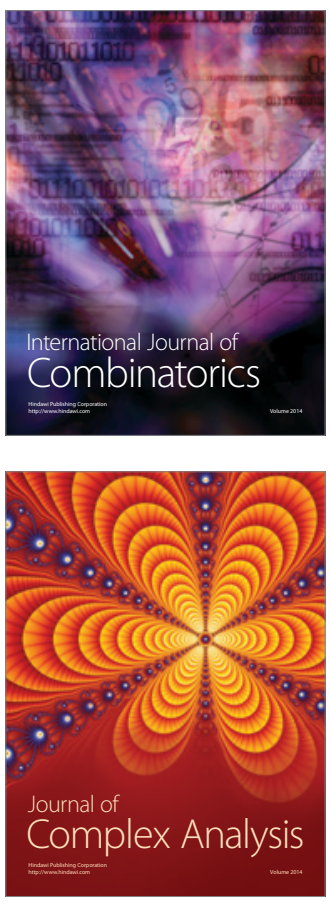

International Journal of

Mathematics and

Mathematical

Sciences
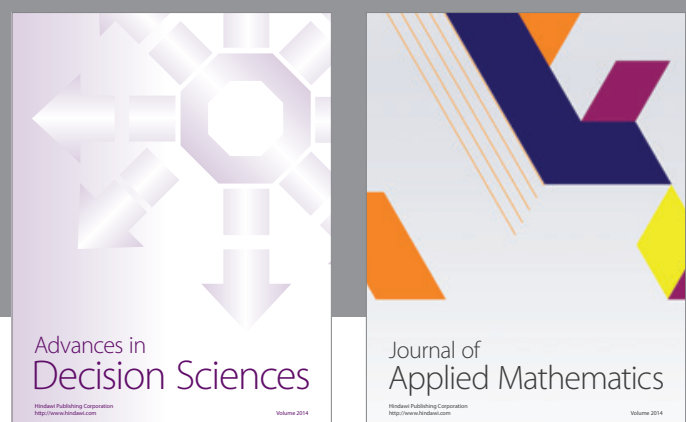

Journal of

Applied Mathematics
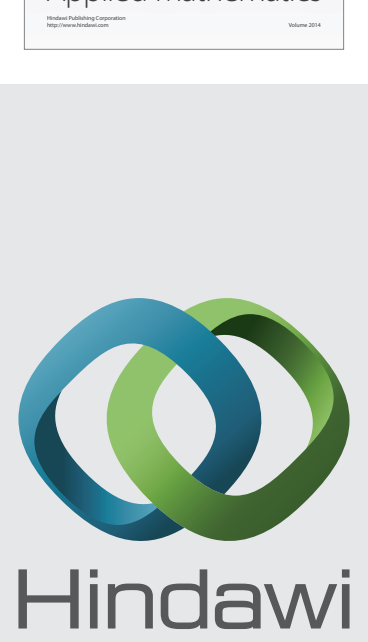

Submit your manuscripts at http://www.hindawi.com
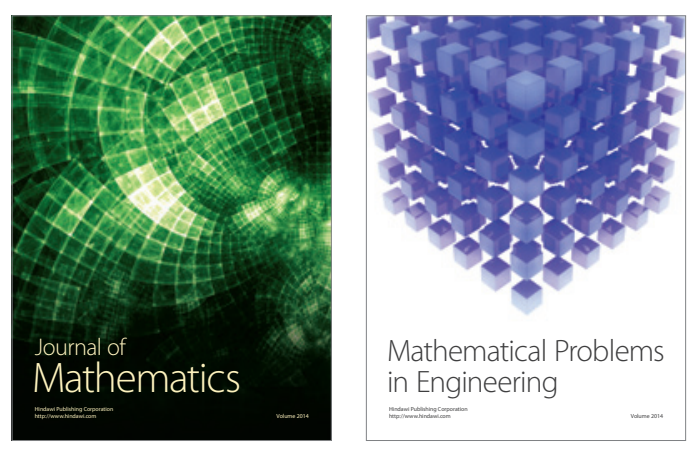

Mathematical Problems in Engineering
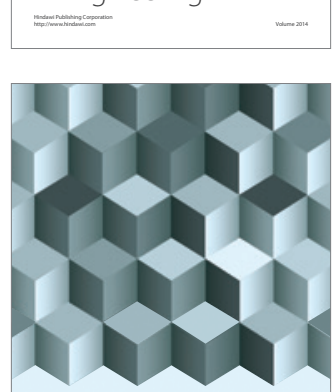

Journal of

Function Spaces
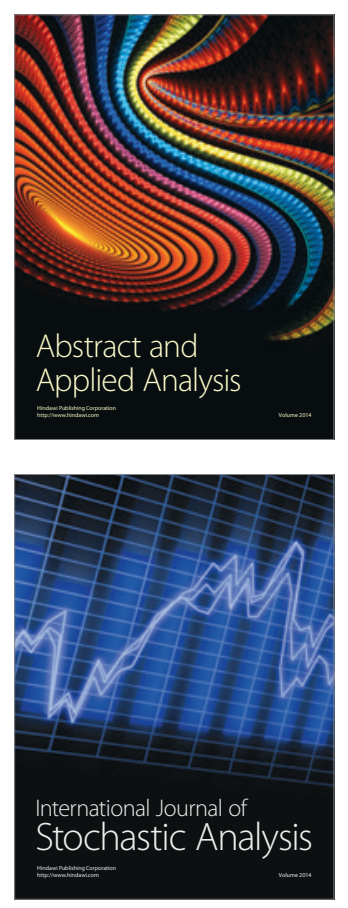

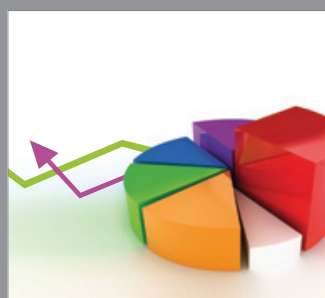

ournal of

Probability and Statistics

Promensencen
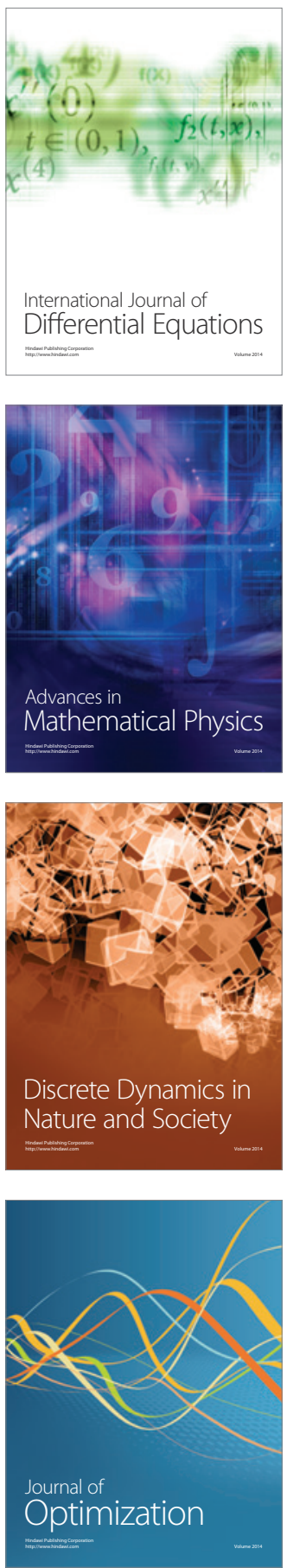